\title{
The Clustering of Acetylcholine Receptors and Formation of Neuromuscular Junctions in Regenerating Mammalian Muscle Grafts
}

\author{
MARK D. WOMBLE \\ Department of Anatomy and Cell Biology, The University of Michigan, \\ Ann Arbor, MI 48109
}

\begin{abstract}
The present investigation was undertaken to study the relationship between acetylcholine receptor (AchR) clustering and endplate formation within regenerating skeletal muscle grafts. Silver staining of nerves was combined with rhodamine-alpha-bungarotoxin labeling of AchR clusters in heterotopic grafts of the rat soleus muscle. Two major graft procedures were used: whole muscle grafts and grafts which lacked the zone of original motor endplates (MEP-less grafts). These categories were subdivided into standard grafts, where subsequent innervation was allowed, and noninnervated grafts, which were experimentally deprived of innervation. Grafting brought about the death and removal of muscle fibers, followed by regeneration of myotubes within surviving basal lamina sheaths. A transient population of small extrajunctinal AchR clusters spontaneously appears shortly after myotube formation in all four muscle graft types. Early myotubes of whole muscle grafts (both innervated and standard grafts, prior to the time of innervation) also develop presumptive secondary synaptic clefts and large, organized aggregations of AchRs at original synaptic sites. At later times, nerves regenerating into standard whole muscle and MEP-less grafts lead to the formation of numerous ectopic endplates. In whole muscle grafts, endplates may also form at original synaptic sites. Functional graft innervation is achieved in whole muscle and MEP-less grafts as early as 20 days postgrafting. The results of this study support the existence of still-unknown factors associated with the original synaptic site which can direct postsynaptic differentiation independent of innervation. They also demonstrate that functional endplates may form in mammalian muscle grafts at both original synaptic sites and ectopic locations, thus indicating that the zone of original synaptic sites is not necessary for the establishment of numerous functional and morphologically well-differentiated endplates.
\end{abstract}

The original synaptic site is known to be involved in the processes of skeletal muscle regeneration and innervation. Several investigators (Sanes et al., 1978; Burden et al., 1979; Bader, 1981) have shown that in regenerating muscle, factors remaining at the original synaptic site are capable of independently directing pre- and postsynaptic differentiation. Thus, in the absence of innervation, high-density acetylcholine receptor (AchR) clusters and secondary synaptic clefts form at original synaptic sites of regenerated frog or rat myotubes (Burden et al., 1979; McMahan et al., 1980; Bader, 1981; Hansen-
Smith, 1983). In amphibian muscle, this synaptic "memory" has been shown to associate specifically with the synaptic portion of the basal lamina (McMahan and Slater, 1984).

Regenerating nerve fibers can easily innervate an intact denervated muscle, at both original synaptic sites (Bennett et al., 1973; Marshall et al., 1977; Gorio et al., 1983) and

The present address for Dr. Mark D. Womble is Department of Physiology, C-240, University of Colorado Health Sciences Center, 4200 E. Ninth Ave., Denver, CO 80262.

Address reprint requests to Dr. B. M. Carlson, Department of Anatomy \& Cell Biology, University of Michigan, Medical Science II, Ann Arbor, MI 48109

Received April 10, 1985. Accepted February 4, 1986. 
ectopic locations (Waerhaug et al., 1977; Lomo and Slater, 1980; Korneliussen and Sommerschild, 1976; Weinberg et al., 1981a, b). Although ectopic endplate formation in regenerating muscle seems to occur (Thompson, 1971, 1974; Gruber et al., 1974; Hakelius, 1979) and muscle grafts become functionally innervated (Thompson, 1971, 1974; Bennett et al., 1974; Hakelius, 1979; Carlson et al., 1981), the importance of the original synaptic site to graft innervation has not been fully examined.

Previous studies (Thompson et al., 1978; Bader, 1980, 1981) using regenerating mammalian muscle grafts in which the zone of original motor endplates (MEPs) was removed at the time of grafting (MEP-less grafts) indicated that the presence of this zone is required for the formation of neuromuscular junctions. Bader $(1980,1981)$ reported that extremely few endplates are formed in MEP-less grafts, despite the presence of normal numbers of nerve fibers. This finding suggests that the original synaptic zone is the major site for endplate formation in mammalian muscle grafts.

In the present study, the relationship between the original synaptic site and muscle regeneration and innervation is examined by using heterotopic whole muscle and MEPless grafts of the rat soleus muscle. Acetylcholine receptor (AchR) clusters were labeled with rhodamine-conjugated alpha-bungarotoxin, in combination with silver impregnation of nerves. The results demonstrate that early in regeneration, AchR clusters develop both specifically, at original synaptic sites of whole muscle grafts, and nonspecifically, in extrajunctional regions of both whole muscle and MEP-less grafts. At later times, in grafts which are allowed to become innervated, functional endplates form at original synaptic sites (in whole muscle grafts only) or at ectopic locations (in both whole muscle and MEP-less grafts). In mature grafts of both types, endplates are numerous and morphologically well differentiated. The results provide support for the presence of synaptic organizing factors located externally to rat muscle myofibers. However, they do not sup. port the idea that the motor endplate region is necessary for functional endplate formation. Some of the results in this paper have been published previously in preliminary forms (Womble, 1982a,b).

\section{MATERIALS AND METHODS Surgical procedures}

All experiments were conducted on male Sprague-Dawley rats (Charles River, Portage, MI) initially weighing $150-200 \mathrm{gm}$. Surgery was done under ether or Metaphane anesthesia.

Heterotopic grafts of the soleus muscle were used in this study. The soleus was chosen because the neuromuscular junctions are confined to the middle third of the muscle (Bennett et al., 1974). This location aids in the identification of original synaptic sites and allows for the production of muscle grafts lacking the zone of original motor endplates (MEP-less grafts).

Due to the lack of a substantial proximal tendon, it is very difficult to return the soleus to its original muscle bed. Therefore, muscles were grafted into the bed of the extensor digitorum longus (EDL) muscle. The tendons of the EDL (approximately $5 \mathrm{~mm}$ in length each) served as proximal and distal attachments for the graft, thus allowing for adjustments for variations in length between whole muscle and the shorter MEP-less grafts, and aiding in graft regeneration and integration.

Four types of soleus muscle grafts were used:

\section{Standard whole muscle grafts}

The left extensor digitorum longus (EDL) muscle was removed and discarded. All vascular, neural, and tendinous connections to the ipsilateral soleus muscle were severed. This entire muscle was transferred to the bed of the ablated EDL muscle, where it was sutured with 7-0 silk surgical thread to the proximal and distal EDL tendons.

\section{Noninnervated whole muscle grafts}

The grafting procedure was identical to that of standard whole muscle grafts, but in addition, at the time of grafting, the ipsilateral sciatic nerve was cut at the level of the sciatic notch and the proximal nerve stump was sutured into the overlying musculature. This prevented innervation of the graft for at least 2 months.

\section{Standard MEP-less grafts}

To produce a motor endplate (MEP)-lacking graft, the soleus muscle was removed and divided transversely into thirds. The central, 
MEP-containing segment was discarded and the two end portions, devoid of MEPs, were sewn together with 7-0 silk surgical thread and sutured to the tendons of the ablated EDL muscle.

\section{Noninnervated MEP-less grafts}

The grafting procedure was identical to that of the standard MEP-less grafts, but in addition, the sciatic nerve was cut as described above.

After grafting, no attempt was made to reestablish neural or vascular connections. The overlying fascia and skin were sutured and animals were housed under standard laboratory conditions.

\section{Histology}

At various times after surgery, ranging from 3 to 60 days, grafted muscles were removed, frozen in dry-ice-cooled 2-methylbutane, and serially sectioned longitudinally on a cryostat at a thickness of $20 \mu \mathrm{m}$. Sections were placed on dry microscope slides, which had previously been treated with a gelatin subbing solution containing 3\% EDTA (MCB Manufacturing Chemists) to retard myofiber contraction, and allowed to dry overnight. Slides were incubated for $1 \mathrm{hr}$ in phosphatebuffered saline (PBS), pH 7.3, containing 1\% bovine serum albumin (BSA) (fraction VI, Sigma Chemical Co.) and $3 \times 10^{-8} \mathrm{M}$ rhodamine-labeled alpha-bungarotoxin (Rh-BTX). Tetramethylrhodamine (Baltimore Biological Laboratory) was conjugated to alpha-bungarotoxin (Biotoxins, Inc.,) by the method of Ravdin and Axelrod (1977). The incubation in Rh-BTX was followed by two 15-min washes in PBS containing 1\% BSA, fixation for $30 \mathrm{~min}$ in $2-3 \%$ paraformaldehyde in $0.1 \mathrm{M}$ phosphate buffer $(\mathrm{pH} 7.3)$, and silver staining by the method of Tsuji and TobinGros (1980). In some cases, the additional step of acetylcholinesterase (AchE) staining with a modification of Karnovsky's procedure (Moody-Corbett and Cohen, 1981) was added between fixation and nerve staining. Preincubation, reaction, and wash solutions for AchE control slides also contained $10^{-5} \mathrm{M}$ Wellcome anti-acetylcholinesterase (Burroughs Wellcome Co.).

\section{Light microscopy}

Tissue sections were examined for fluorescence using a Leitz Orhtoplan microscope equipped with a xenon lamp and Ploempak filter cubes for epifluorescence. The excitation filter was $530-560 \mathrm{~nm}$ and the fluorescence barrier filter was $580 \mathrm{~nm}$.

\section{Electron microscopy}

Muscles were removed, pinned at resting length, and fixed overnight in 3\% glutaraldehyde in $0.1 \mathrm{M}$ phosphate buffer $(\mathrm{pH}$ 7.3). After 3 rinses in $0.1 \mathrm{M}$ phosphate buffer, the muscle was embedded in $6 \%$ warm gelatin, cooled, and sliced longitudinally at $230 \mu \mathrm{m}$ on a Smith-Farquar tissue chopper. The slices were stained for acetylcholinesterase activity (Karnovsky, 1964) and reactive areas were cut out, postfixed in $1 \%$ osmium tetroxide in phosphate buffer containing $0.5 \mathrm{mg} / \mathrm{ml}$ ruthenium red (Luft, 1971), and embedded in Epon 812 resin. Pale gold sections were cut, stained with uranyl acetate and lead citrate, and examined on a Siemens 101 electron microscope.

\section{Determination of Ach $R$ cluster numbers and sizes}

The number of receptor clusters was determined by examining approximately every fifth section. All clusters within the section were counted, the section counts were averaged to give a mean cluster number per section, and this was multiplied by the number of sections to yield the total number of clusters of each type for that muscle. The results from at least three muscles per time point were taken to give an average cluster number per muscle.

The labeling technique employed here reveals high-density receptor clusters, as small as $3.0 \mu \mathrm{m}$ in diameter, but is not sensitive enough to identify areas of diffusely distributed AchRs. Cluster type was determined on the basis of morphology, size, and location within the graft. Endplates were defined as any definitive cluster of AchR-specific fluorescence contacted by a nerve fiber. Receptor cluster sizes were determined using an ocular micrometer to obtain direct measurements of cluster length along the long axis of the myofiber. All measurements are given with the standard error of the mean.

\section{Contractile activity}

The presence of nerve-evoked contractile activity was determined by direct nerve 
trunk stimulation, while muscle activity was monitored both visually and electrically. The animal was anesthetized with ether, and the muscle graft with its nerve supply was identified. All surrounding muscles were denervated, taking care not to damage the nerve branches to the graft. The distal tendinous end of the graft was cut and two fine-wire recording electrodes were inserted into the muscle. The nerve to the graft (the common peroneal nerve) was severed as far proximally into the thigh region as possible, yielding a nerve trunk of $2-3 \mathrm{~cm}$. As the nerve was cut, the graft was watched for any contractile response. Nerve stimulations, via two fine-wire stimulating electrodes, were done at $20 \mathrm{~V}$ at a frequency of $50 \mathrm{~Hz}$ and a duration of $0.5 \mathrm{~ms}$. At each stimulation, the muscle activity was monitored both for an oscilloscope-displayed electrical response from the recording electrodes and visually for gross signs of muscle contraction. To control for the possibility of current spread down the nerve to the muscle in some grafts (three standard whole muscle and MEP-less grafts each at 30 and 45 days), response recordings were done before and after a nerve crush (Gerding et al., 1977; Robbins et al., 1977). The nerve to the graft was stimulated, and a response was confirmed from the muscle. The nerve was then crushed and again stimulated. No muscle response was obtained when the nerve was stimulated distal to the crush. A response, identical to that seen before the nerve crush, was always seen when the nerve was stimulated at a point closer to the muscle than the site of the nerve crush. This indicates that the muscle activity recorded upon nerve stimulation is due to a true nerveevoked contractile response and not to a nonspecific spread of current down the nerve trunk to the muscle.

\section{RESULTS}

The free grafting of a skeletal muscle leads to myofiber degeneration and removal by phagocytic cells (Hansen-Smith and Carlson, 1979; Carlson and Faulkner, 1983), while leaving basal lamina sheaths intact (HansenSmith and Carlson, 1979; Marshall et al., 1977; Sanes et al., 1978). Inside these sheaths, muscle satellite cells proliferate and fuse to form new muscle fibers (Snow, 1978). This sequence occurs in both whole muscle and MEP-less grafts.

\section{Loss of original AchRs}

To study the appearance of regenerated AchR clusters in whole muscle grafts, it was important to demonstrate that original endplate receptor aggregates did not survive the processes of degeneration and phagocytosis. Therefore, regions of early (3-day) whole muscle grafts where phagocytosis was complete but where myotube formation had not yet begun were examined.

The position of the original synaptic site was located by staining for residual acetylcholinesterase (AchE) activity. This enzyme is highly concentrated at the neuromuscular junction, where it remains after phagocytosis of the myofiber (Marshall et al., 1977; J. Harris, 1981). Examination of four muscles produced no AchR-specific fluorescence at any of 33 junctional AchE-positive sites. Thus, original synaptic AchR aggregates did not survive myofiber phagocytosis in whole muscle grafts.

Removal of the endplate zone at the time of muscle grafting produces an MEP-less graft. Two tests were used to demonstrate removal of the endplate zone. In one, at the time of removal, the entire central MEP-containing muscle segment was reacted for AchE activity. This produced a visible reaction product which could be followed around the perimeter of the muscle section. Secondly, five noninnervated MEP-less grafts, ages 1030 days postgrafting, were sectioned and stained for residual junctional AchE activity. Both series of experiments show that in all cases the vast majority of original motor endplates are removed at the time of grafting. Although evidence of a few remaining original synaptic sites was found in some MEPless grafts, the number of such sites is very low (no more than 30-50 per muscle graft) and is not considered significant in comparison to the approximately 3,400 endplates (Bader, 1980) of a normal soleus muscle. The few remaining original sites, if present, are always confined to a limited area near the junction of the two muscle graft halves.

\section{Appearance of AchR clusters}

Three days after grafting, no new myotubes are seen. By 5 days, however, active myotube formation has begun in both whole muscle and MEP-less grafts; and at 7-10 days, well before the arrival of nerve fibers, 

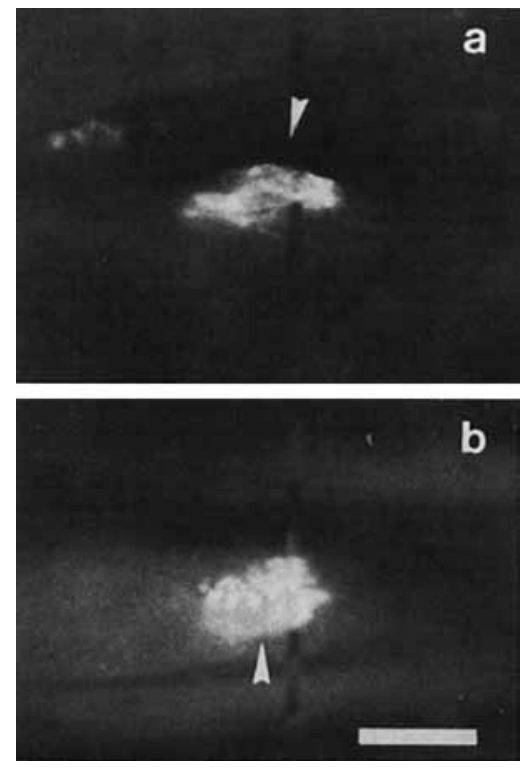

Fig. 1. Extrajunctional AchR clusters (arrowheads) from regenerating muscle grafts. a. Cluster from a 7-day standard MEP-less graft b. Cluster from a 20-day noninnervated whole-muscle graft. Note the diffuse, ill-defined edges of the fluorescence pattern. $\mathrm{Bar}=10 \mu \mathrm{m}$.

myotubes are found throughout the grafts. Newly regenerated myotubes of both whole muscle and MEP-less, noninnervated and standard grafts (prior to innervation) develop extrajunctional AchR clusters.

Extrajunctional clusters (Fig. 1) are found distributed throughout the entire muscle. They have diffuse, ill-defined edges that fade into the background fluorescence of the myofiber. Internally, they are made up of lines or dots of fluorescence (Fig. 1a), similar to other nonsynaptic receptor clusters (Axelrod et al., 1976; Anderson and Cohen, 1977; Kidokoro et al., 1980; Ishikawa and Shimada, 1982).

Extrajunctional clusters are found at 5 days postgrafting, within $48 \mathrm{hr}$ after the start of myotube formation. They increase rapidly in number (Fig. 2) and size, growing in whole muscle regenerates from a length of $12.9 \pm$ $0.4 \mu \mathrm{m}$ at 5 days to $17.1 \pm 0.5 \mu \mathrm{m}$ at day $\overline{7}$. Beyond this time, cluster size declines significantly, to $12.6 \pm 0.5 \mu \mathrm{m}$ at 10 days, and remains at a baseline size of approximately $12 \mu \mathrm{m}$ up to 60 days postgrafting. The decrease in cluster size is paralleled by a drastic decline in number (Fig. 2), so that by 15 days practically all extrajunctional clusters have disappeared. This rapid fluctuation in cluster number appears to be spontaneous and intrinsic to the early phases of muscle regeneration, occurring in both standard whole muscle and MEP-less grafts prior to innervation, and noninnervated whole muscle and MEP-less grafts (data not shown).

Another AchR cluster type, found only in whole muscle grafts (both noninnervated and standard, prior to innervation), is the endplatelike (EP-like) cluster (Fig. 3). EP-like clusters are large, with well-defined edges, and display within the cluster a branching and anastomosing pattern of fluorescence similar in morphology to normal soleus muscle endplates (Steinbach, 1981). They are made up of small "speckles" or dots of fluorescence (Fig. 3), as reported for other junctional AchR clusters (Steinbach, 1981; Ishikawa and Shimada, 1982). EP-like clusters are distributed solely within the central third of the graft, in the same manner as endplates of a normal rat soleus muscle (Bennett et al., 1974). They are not found in MEPless grafts.

EP-like clusters are found in noninnervated and standard whole-muscle grafts on day 5 , within $48 \mathrm{hr}$ of initial myotube formation. They increase rapidly in number (Fig. 4). Significantly, even at their earliest appearance on the most immature myotubes, these clusters fully display the characteristic EP-like branching morphology. Averaging $28.7 \pm 0.7 \mu \mathrm{m}$ length in 5-day grafts, they are more than twice the size of extrajunctional clusters at this time. Myotube growth is accompanied by an increase in the size of EP-like clusters, attaining a length of $34.6 \pm$ $0.9 \mu \mathrm{m}$ in 15-day grafts. From 15 to 60 days, EP-like clusters are not significantly different in size from the control soleus muscle endplate length of $37.1 \pm 1.5 \mu \mathrm{m}$. Beyond 30 days, there is a decline in cluster number in both noninnervated and standard grafts (Fig. 4), although some remain for at least 60 days after grafting. In well-innervated 60-day standard whole muscle grafts, the few remaining EP-like clusters are associated only with very thin, apparently noninnervated, myofibers.

Staining for residual junctional AchE activity to mark the original synaptic sites in conjunction with Rh-BTX labeling reveals that the EP-like receptor clusters develop 

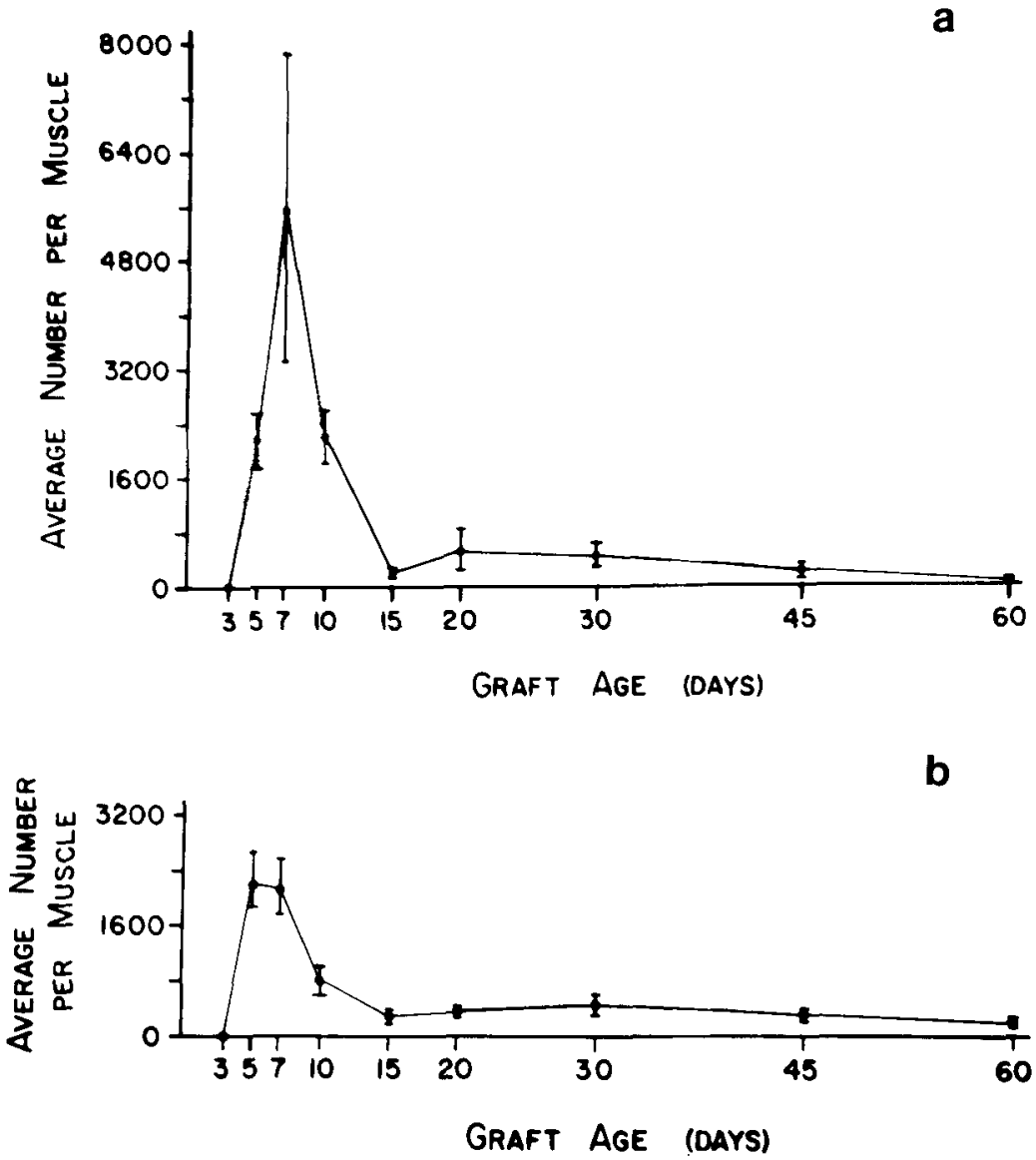

Fig. 2. Number of extrajunctional receptor clusters per muscle for standard whole-muscle (a) and MEP-less (b) grafts. Results from noninnervated grafts were iden-

solely at former synaptic sites. Examination of 10-30-day noninnervated whole-muscle grafts demonstrates that, in all cases, the fluorescent EP-like clusters correspond exactly in size, shape, and location to areas of junctional AchE staining (Fig. 5). Incubation of control sections in anti-AchE totally eliminates all esterase staining at these EP-like sites, confirming that AchE was the enzyme acting to produce the staining.

Muscle cell membrane invaginations, similar to secondary synaptic clefts of normal neuromuscular junctions, are also specifically associated with sites of residual junctional AchE activity (Fig. 6). These clefts are seen in noninnervated whole-muscle grafts within days of myotube formation. tical (data not shown). Each point represents average ( $t$ S.E.M.) of three to ten muscles. No clusters were found in 3-day grafts.

\section{Innervation of whole-muscle grafts}

Regenerating nerve fibers first enter standard whole-muscle grafts beginning approximately 10-15 days postgrafting, where they grow among the developing myofibers. Endplates form at either ectopic locations or by the apparent reinnervation of original synaptic sites, as marked by the EP-like receptor clusters. The site of initial nerve entry determines the number of endplates in these two categories: nerves which enter the muscle centrally, close to the original synaptic zone, will predominantly form endplates in this region, while numerous ectopic endplates are formed by nerves confined to more proximal muscle areas. 

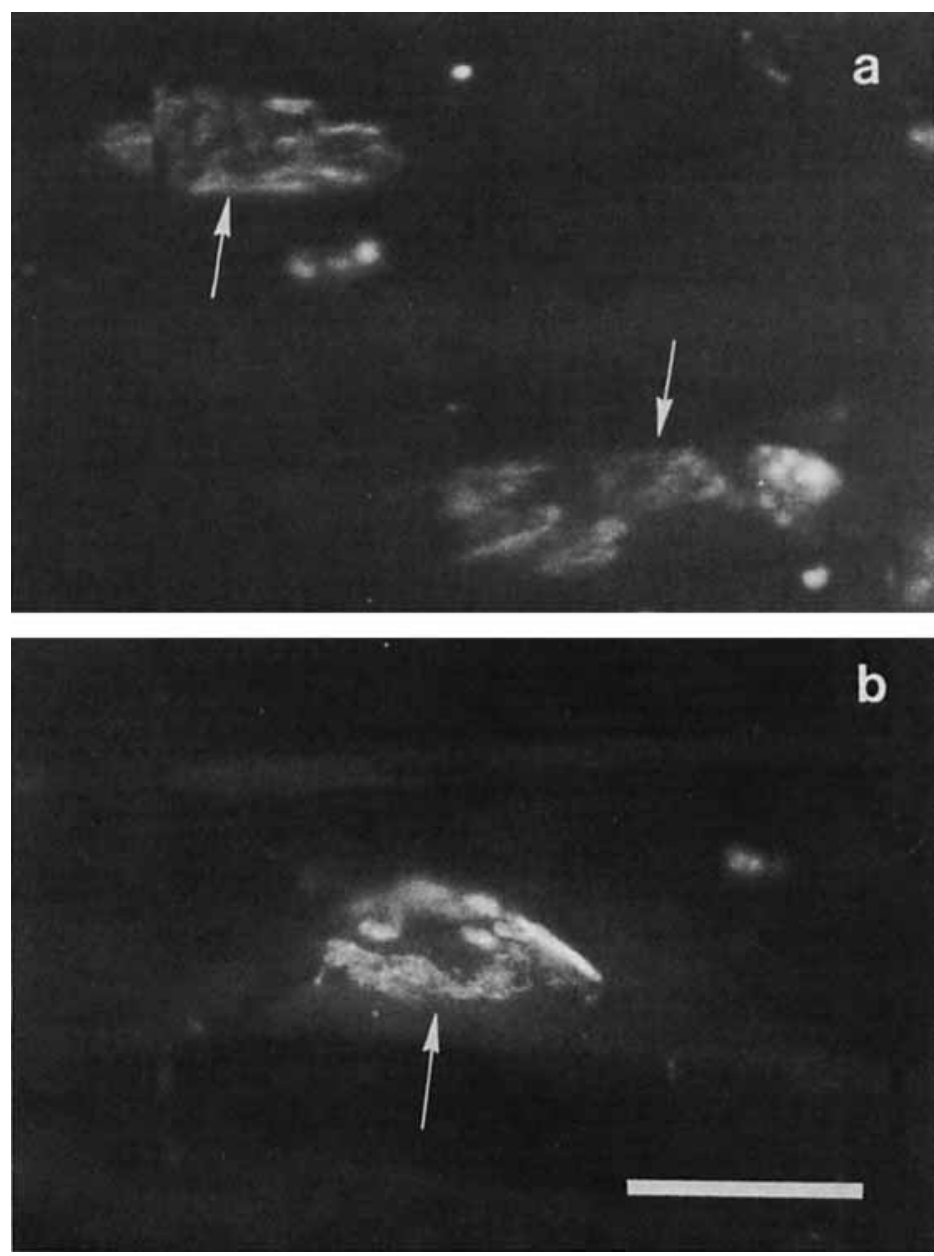

Fig. 3. Endplatelike receptor clusters (arrows) from whole muscle grafts. Note the branching pattern and well-defined edges of fluorescence. a. Cluster from a 7 - day standard graft. b. Cluster from a 45 -day noninnervated graft. Bar $=20 \mu \mathrm{m}$
Early ectopic endplates are small and poorly developed. At maturity (45-60 days postgrafting), they are similar in size (average length of $40.7 \pm 1.7 \mu \mathrm{m}$ ) and morphology to normal soleus muscle neuromuscular junctions.

Centrally, direct innervation of some EP. like clusters was observed (Fig. 7). After a small inital contact, the nerve terminal comes to cover almost the entire area of clustered receptors. These endplates reach a mature length of $44.5 \pm 4.5 \mu \mathrm{m}$ and are morphologically identical to control soleus muscle endplates.
Functional innervation of whole muscle grafts was first observed at 20 days, when stimulation of the regenerated nerve trunk produced a visible contraction in two out of three muscles. Endplate formation proceeds at a steady rate up to about 45 days postgrafting, after which the total number of endplates per muscle levels off, reaching 1442 $\pm 347(\mathrm{n}=10)$ at 60 days (Fig. 8). All 11 mature (45-60-day) grafts tested physiologically demonstrate at least some degree of functional innervation. Histological examination reveals that some of these muscles are predominantly innervated centrally, pre- 

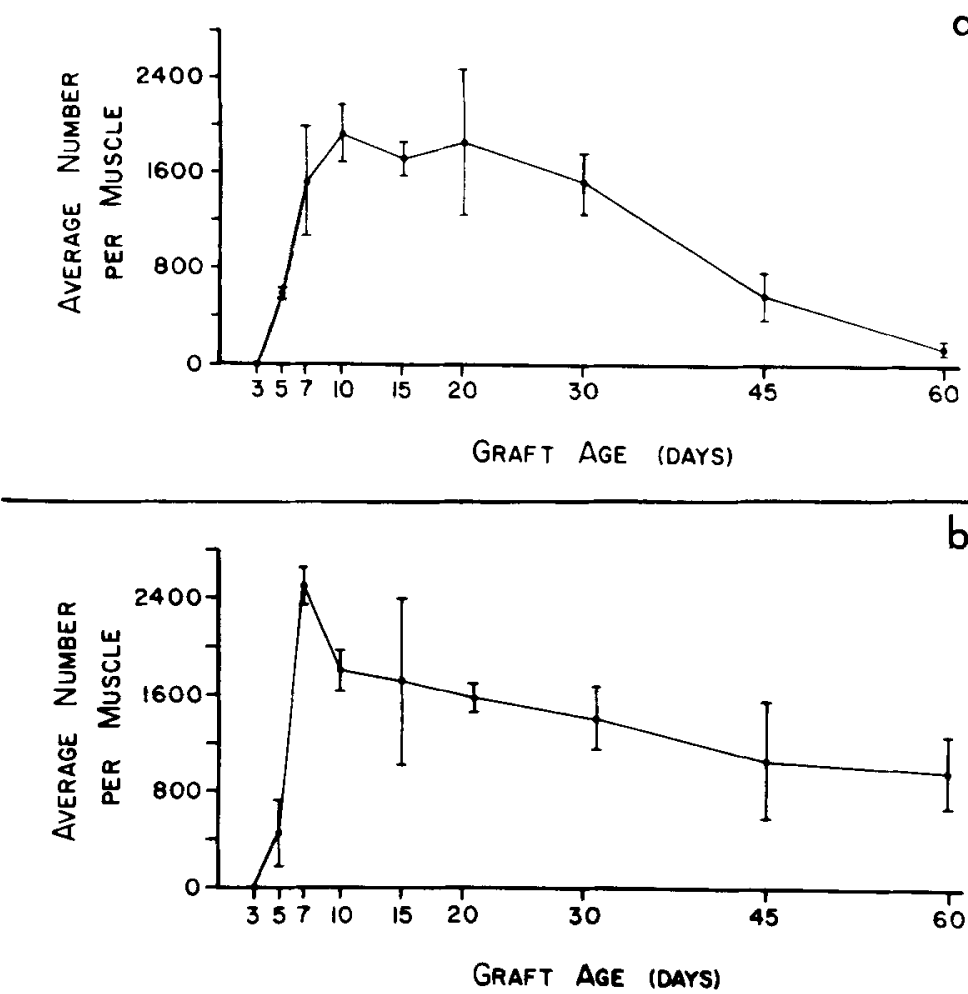

Fig. 4. Number of EP-like AchR clusters per muscle for (a) innervated and (b) noninnervated whole-muscle

sumably at original synaptic sites. In others, endplates are found almost exclusively at ectopic locations.

\section{Innervation of MEP-less grafts}

Endplate formation in standard MEP-less grafts also first begins at about days $10-15$, soon after initial nerve entry, and proceeds at a steady rate through 60 days postoperatively (Fig. 8). Since original synaptic sites are removed at the time of grafting, new neuromuscular junctions are formed exclusively at ectopic locations. Regenerating axons encounter myofibers, leading to specific AchR accumulations at sites of nerve-muscle contact. These sites develop into large (30$40 \mu \mathrm{m}$ in length), morphologically normalappearing endplates (Fig. 9), with strong acetylcholinesterase activity. Large numbers of endplates are found in 60-day MEP-less grafts, averaging $1,663 \pm 244$ endplates per graft $(n=15)$ (Fig. 8). This number is not statistically different from the number of grafts. Each point represents the average ( \pm S.E.M.) of three to ten muscles. No clusters were found at 3 days. endplates found in 60-day standard wholemuscle grafts.

Nerve stimulation tests were also performed on MEP-less grafts 10-60 days postoperatively. Functional innervation was first found at 20 days, when one of two muscles responded to nerve stimulation. From a total of 28 mature grafts ( 45 and 60 days postgrafting) examined histologically, only one contained no endplates. All 15 muscles tested from this group showed signs of nerve-evoked responses. Thus by 45-60 days, almost all MEP-less grafts are well innervated and able to respond to nerve stimulation.

\section{DISCUSSTON}

The present study examines the clustering of AchRs and the innervation of regenerating whole muscle and MEP-less skeletal muscle grafts. In whole-muscle grafts, postsynaptic endplate specializations (such as organized AchR clusters and presumptive secondary synaptic clefts) form at original synaptic sites 

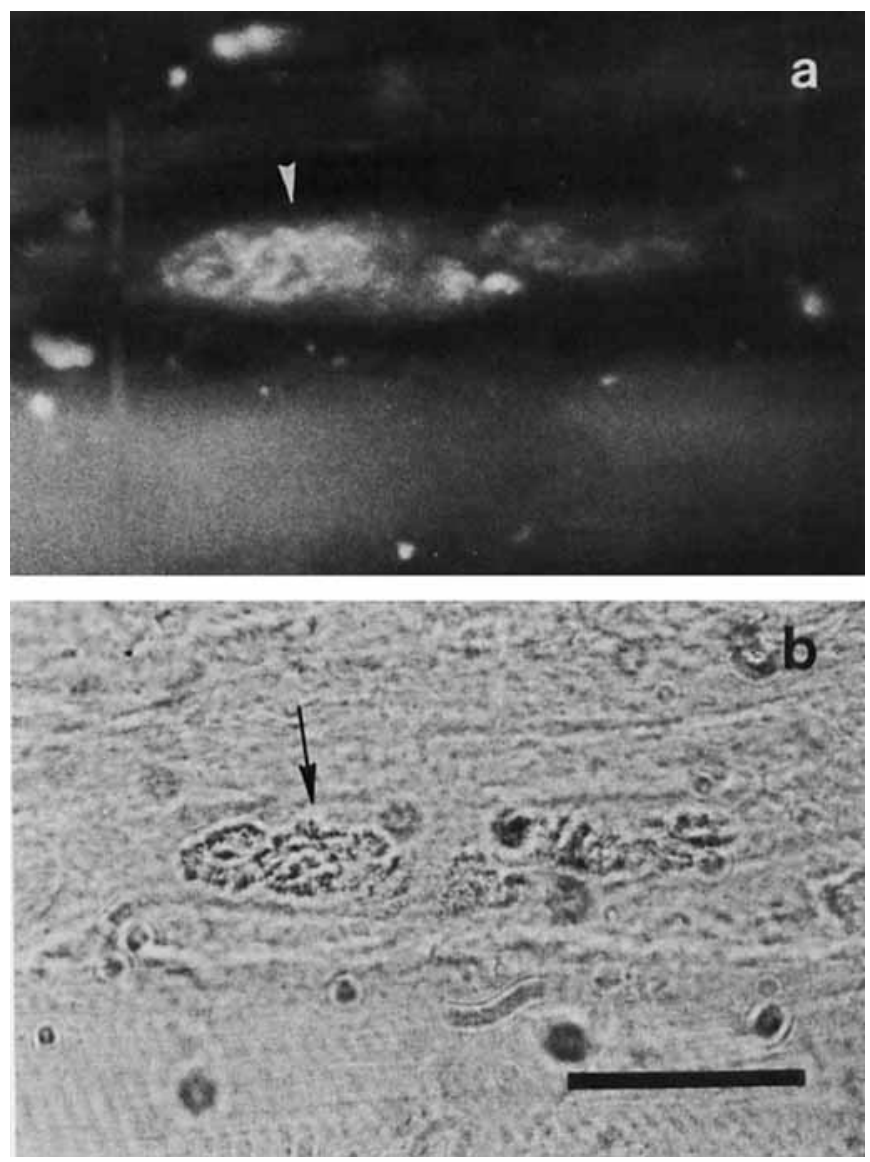

Fig. 5. A fluorescently labeled EP-like AchR cluster (arrowhead in a) colocalized with remaining junctional AchE staining (arrow in b). Note the very close correspondence in branching pattern between the AchE stain ing (marking the original synpatic site) and that of the

in the absence of innervation. Subsequent functional endplate formation may occur at these sites as well as at ectopic locations. MEP-less grafts receive abundant ectopic innervation. Both graft types display nerveevoked activity as early as 20 days postgrafting.

\section{AchR Clustering at the original synaptic site}

Grafting of the rat soleus muscle leads to degeneration and phagocytosis of the myofibers (Hansen-Smith and Carlson, 1979; Carlson and Faulkner, 1983). This process brings about, in whole muscle grafts, the loss of all junctional AchR aggregates, as determined by fluorescent bungarotoxin labeling. A sim-
EP-like cluster. The AchE staining is intentionally light in order not to obscure the underlying AchR fluorescent label. Taken from a 10-day noninnervated whole-muscle graft. Bar $=20 \mu \mathrm{m}$.

ilar result was obtained with fluorescent AchR labeling by Harris (1982) in degenerating rat muscle, although in degenerating frog muscle the more sensitive probe of radioactively labeled bungarotoxin reveals an approximate $10 \%$ retention level of AchRs at the original synaptic site (Burden et al., 1979; McMahan and Slater, 1984).

In both noninnervated and standard (prior to innervation) whole muscle grafts, highdensity AchR clusters (the EP-like clusters) are found at original synaptic sites, confirming earlier results from frog (Burden et al., 1979; McMahan and Slater, 1984) and rat muscle (Bader, 1981; Hansen-Smith, 1983). Since EP-like clusters do not appear at other positions within the graft, their development 


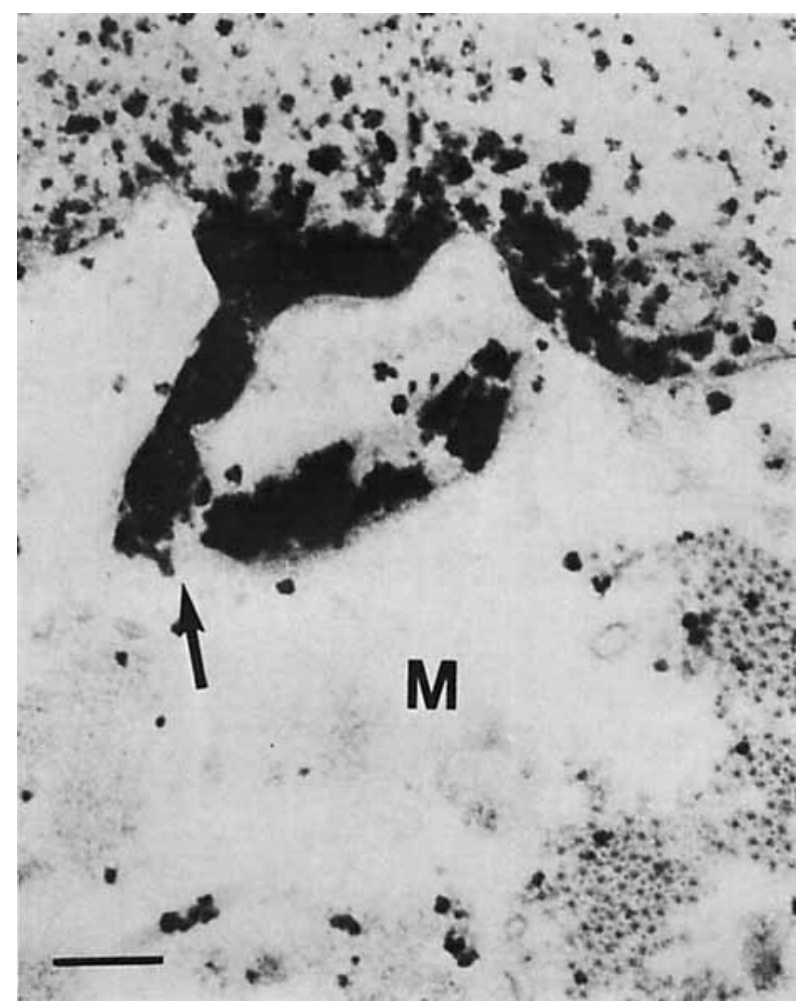

Fig. 6. Electron micrograph of a 7-day noninnervated whole-muscle graft showing a newly regenerated myotube $(\mathrm{M})$ containing small bundles of myofibrils cut in cross section (lower right). Staining for residual junctional AchE activity (black reaction product) identifies this region as an original synaptic site. Associated with this region is a deep surface invagination (arrow) filled with AchE reaction product. The invagination resembles the secondary synaptic cleft of the normal neuromuscular junction. Bar $=1 \mu \mathrm{m}$. is apparently dependent on information remaining at the original synaptic site after myofiber phagocytosis. This suggests the presence of factors external to the muscle cell capable of guiding the localization and organization of AchRs at former junctional sites (Burden et al., 1979).

These factors are apparently highly organized at the former synaptic site, as revealed in the precise pattern of arborization shown by EP-like receptor clusters. The long-term retention (for as long as 60 days on noninnervated myofibers) of EP-like clusters implies that these factors are also very stable. Alternatively, this cluster longevity may simply have been due to the inherent stability of high density junctional receptor aggregates (Hartzell and Fambrough, 1972; Frank et al., 1975; Porter and Barnard, 1975).

Beyond 30 days postgrafting, there is a decline in the number of EP-like clusters in both standard and noninnervated wholemuscle grafts. In chronically noninnervated grafts, this decline is less pronounced and may be related to muscle atrophy. These grafts have greatly reduced whole-muscle size and weight, as well as extremely thin myofibers. In long-term standard grafts, the process of muscle innervation may lead to the loss of EP-like clusters by one of two possible mechanisms. First, the cluster could be innervated directly by a regenerating nerve fiber, converting it into an endplate. Secondly, the myofiber may become innervated at an ectopic location, leading over a period of time to the disappearance of noninnervated EP-like cluster due to myofiber activity generated through the ectopic endplate (Frank et al., 1975).

Muscle-cell membrane invaginations closely resembling normal endplate secondary synaptic clefts also appear at original 

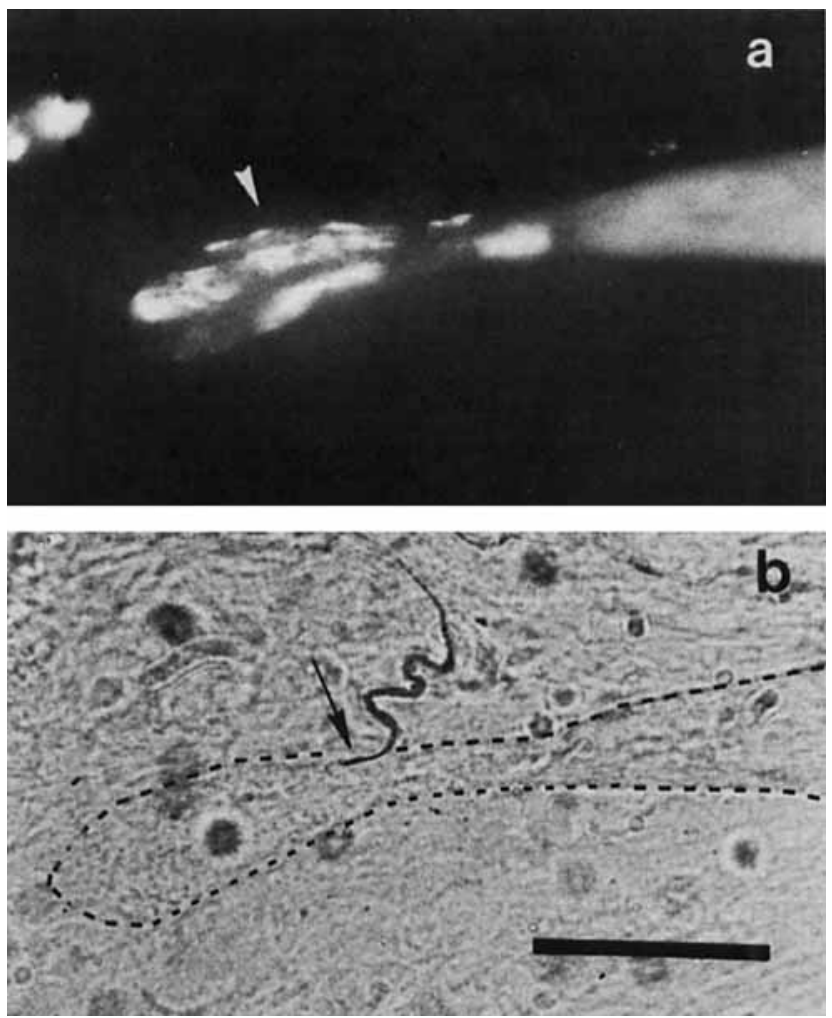

Fig. 7. Innervation of an EP-like AchR cluster by a regenerating nerve fiber, taken from a 30 -day standard whole-muscle graft. a. A fluorescent EP-like cluster (ar- rowhead) is shown. $b$. There is specific contact of this site by a returning nerve fiber (arrow). The myofiber is delineated by the dotted line. Bar $=20 \mu \mathrm{m}$. synaptic sites of whole-muscle grafts. Similar structures have been reported in regenerating frog (Burden et al., 1979; McMahan et al., 1980) and rat (Bader, 1981; HansenSmith, 1983; Sanes et al., 1984) muscles.

Thus, the remaining information associ. ated with the former synaptic region is able to guide several forms of postsynaptic differentiation, independent of innervation, including organized AchR accumulation and synaptic cleft formation. In frog muscle, this information has been shown to be specifically associated with the junctional portion of the basal lamina sheath (Sanes et al., 1978; Burden et al., 1979; McMahan and Slater, 1984). In both regenerating frog and rat muscle, the action of the "guide" may simply be to trig. ger a preprogramed mechanism within the muscle cell similar to that seen in cultured Xenopus myotomal cells, where AchR clusters and surface invaginations develop at the site of interaction with latex beads (Peng and Cheng, 1982).

\section{Extrajunctional AchR clusters}

The regenerative phase of a rat muscle graft, with its rapid myogenic cell proliferation and fusion to produce myotubes, is very similar to embryonic muscle development. One difference is that regenerated myotubes of both early whole muscle and MEP-less grafts develop numerous extrajunctional receptor clusters. While fetal myofibers do show evidence of extrajunctional receptors, these receptors are not found organized into clusters. However, extrajunctional clusters are produced by embryonic rat myotubes in vivo after denervation or treatment with tetrodotoxin to prevent nerve-evoked muscle activity (Braithwaite and Harris, 1979; A. Harris, 1981; Slater, 1982; Ziskind-Conhaim and Bennett, 1982). Under these conditions, nu- 


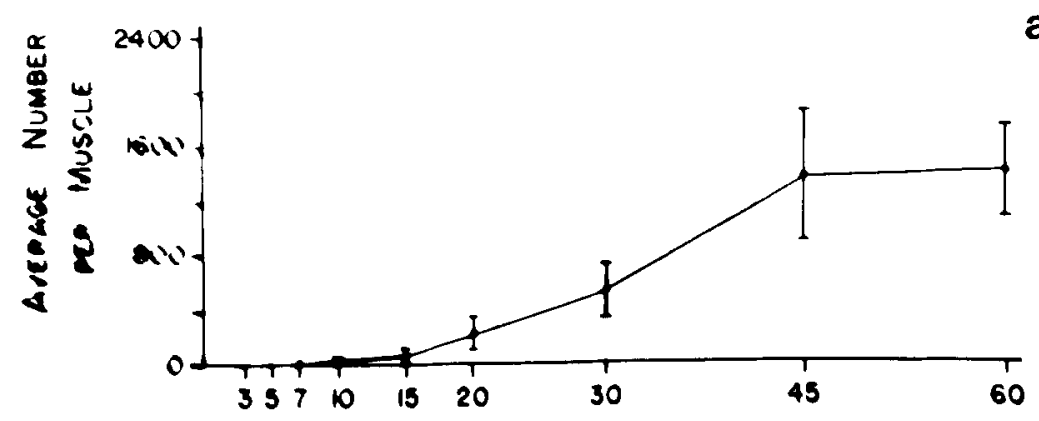

Graft age (Days)

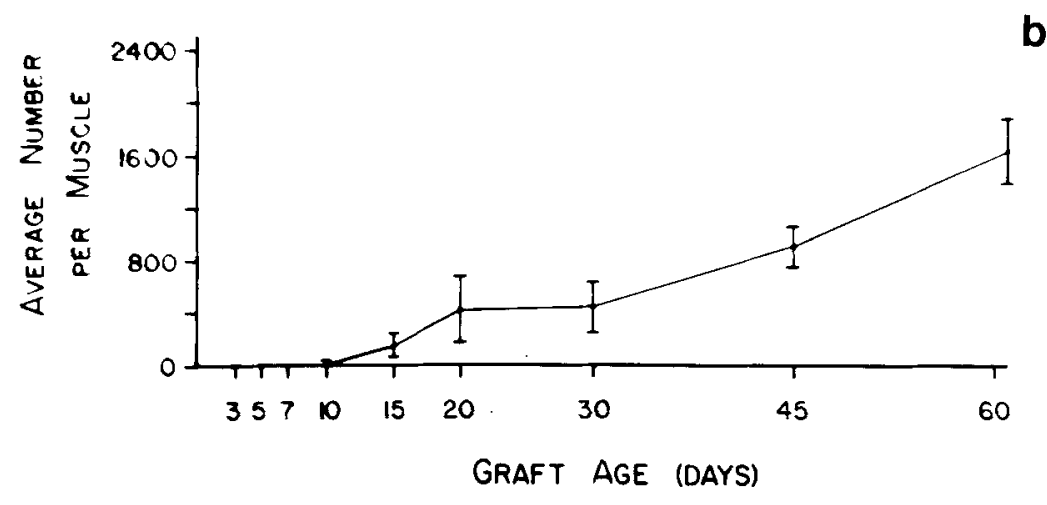

Fig. 8. Number of endplates per muscle in standard grafts. Each point represents the average ( \pm S.E.M.) of three to ten muscles. No endplates were found prior to 10 days. a. Whole-muscle grafts. b. MEP-less grafts. merous extrajunctional clusters appear, approximately $5-22 \mu \mathrm{m}$ in size, comparable to those seen in the present study. Thus, cluster formation in early muscle regenerates may be a response to aneural conditions.

The subsequent rapid decline in extrajunctional cluster number is nerve independent, occurring in both noninnervated and standard (prior to the time of innervation) whole muscle and MEP-less grafts. Receptor cluster loss may be due to muscle fiber fibrillation, since spontaneous contraction of embryonic myotubes grown in aneural cultures is known to cause the breakup and disappearance of nonsynaptic receptor clusters (Prives et al., 1976; Spector and Prives, 1977).

\section{Innervation of grafts}

Muscle-graft innervation begins approximately $10-15$ days postgrafting. In standard whole-muscle grafts, some original synaptic sites may be innervated as regenerating nerve fibers contact EP-like receptor clus- ters. This conclusion was reached from observations of nerve fibers approaching or just contacting EP-like clusters, and from the fact that no signs of apparent de novo endplate formation were seen within this zone. However, the possibility that endplates develope within this zone at positions other than at the original synaptic site cannot be completely excluded.

It is well known that after nerve injury nerves regenerating into an uninjured muscle will preferentially reinnervate the original synaptic site (Iwayama, 1969; Bennett et al., 1973; Jansen and van Essen, 1975; Gorio et al., 1983). A returning nerve will also selectively reinnervate these sites after muscle damage, with (Marshall et al., 1977; Vedung, 1983) or without (Sanes et al., 1978) subsequent muscle-fiber regeneration. Thus, it is not surprising to find innervation of EP-like receptor clusters.

Ectopic innervation also occurs in whole muscle, as well as in MEP-less standard 

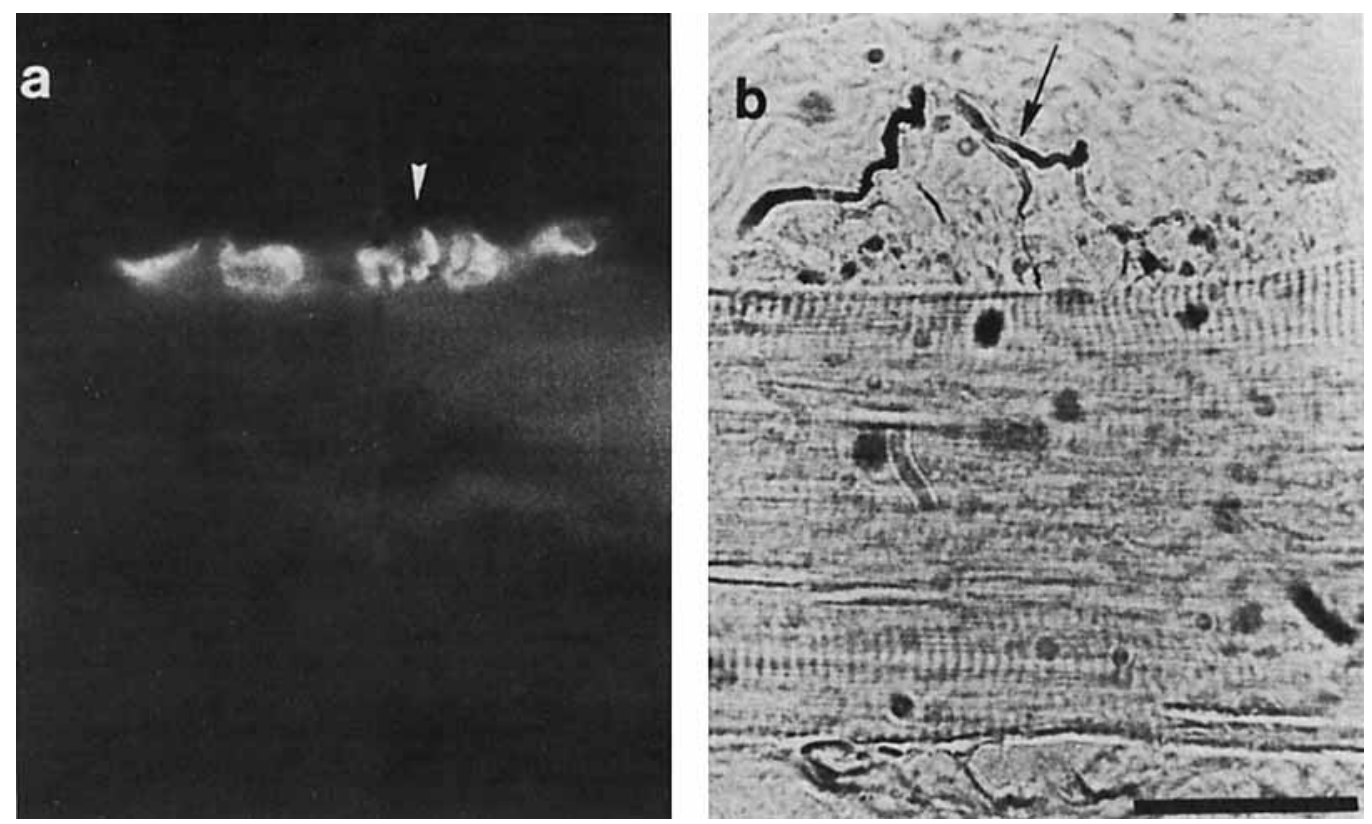

Fig. 9. Endplate from a 60-day MEP-less graft with a nerve fiber (arrow) contacting a fluorescently-labeled AchR cluster (arrowhead). Note the large-diameter myofiber associated with this well-differentiated endplate. Bar $=20 \mu \mathrm{m}$.

grafts. These endplates begin as small areas of AchR clustering at nerve-muscle contact sites before maturing into morphologically normal-appearing endplates. This pattern is similar to that seen during embryonic endplate formation (Braithwaite and Harris, 1979; Dennis et al., 1981; Steinbach, 1981), endplate formation in nerve-muscle cocultures (Anderson and Cohen, 1977; Kidokoro and Yeh, 1982; Moody-Corbett and Cohen, 1982), and during ectopic endplate formation in adult muscle (Waerhaug et al., 1977; Lomo and Slater, 1980; Weinberg et al., 1981a,b). Ectopic innervation of regenerated muscle has been reported in rat (Bennett et al., 1974; Gruber et al., 1974; Hansen-Smith, 1983), cat (Hakelius, 1979), and dog (Thompson, 1971, 1974) muscle.

Nerve-evoked muscle activity is first detected in both whole-muscle and MEP-less standard grafts at 20 days postgrafting. This timing is similar to other studies of muscle. graft innervation (Bennett et al., 1974; Carlson et al., 1981). By 45-60 days, all grafts tested (11 whole muscle and 15 MEP-less grafts) showed signs of functional innervation.
In whole-muscle grafts, the total number of endplates per muscle reached the level of $1,442 \pm 347$ endplates $(n=10)$ at 60 days postgrafting. Although well below the number of 3,412 endplates in a normal soleus muscle reported by Bader (1980), it is in agreement with the number of endplates per muscle obtained by him in whole soleus muscle grafts. In MEP-less grafts, the number of endplates per muscle increases steadily, reaching the level of $1,633 \pm 244$ at 60 days ( $n=15$ ), not statistically different from the number of endplates in whole-muscle grafts.

The MEP-less graft results, however, contradict previous findings (Thompson et al., 1978; Bader, 1980, 1981). Bader, also using MEP-less rat soleus muscle grafts, reported that very few endplates were formed, as determined by combined nerve and AchE staining. $\mathrm{He}$ found an average number of endplates per muscle of $46 \pm 22$ (S.D.) at 40 days $(n=5)$ and $117 \pm 61$ (S.D.) at 60 days $(\mathrm{n}=7)$ in two different studies. By morphological, physiological, histochemical, and biochemical criteria, the muscles he tested were essentially totally denervated, despite the fact that nerves were present at normal lev- 
els within the grafts at earlier time points (up to approximately 30 days). The few endplates present did show normal pre- and postsynaptic ultrastructural differentiation.

Although in the present study, small numbers of original synaptic sites may remain in some MEP-less grafts after the removal of the motor endplate zone, their numbers are considered insignificant when compared to the large number of endplates found in mature MEP-less grafts. Thus, reinnervation of former synaptic sites cannot explain the differences between present and previous MEPless graft studies. The differences may lie in variations in surgical procedures.

Located at the original synaptic site are unknown factors capable of directing both pre- and postsynaptic differentiation following nerve-muscle regeneration (Sanes et al., 1978; Burden et al., 1979; Bader, 1981). The present study confirms that these factors can produce organized AchR clusters and presumptive secondary synaptic clefts on regenerating rat skeletal muscle fibers. The original synaptic site is also a possible site of muscle-graft innervation. Based on previous MEP-less graft work, it had been postulated that the zone of original synaptic sites is required for the innervation of regenerating mammalian muscle grafts (Bader, 1980, 1981). This suggestion is not supported by the present investigation. Endplates are found to form readily at ectopic locations in both whole-muscle and MEP-less muscle grafts. Thus, it is concluded that the zone or original synaptic sites is not required for the functional innervation of regenerating mammalian skeletal muscle grafts.

\section{LITERATURE CITED}

Anderson, M.J., and M.W. Cohen 1977 Nerve-induced and spontaneous redistribution of acetylcholine receptors on cultured muscle cells. J Physiol (Lond.), 268:757-773.

Axelrod, D., P. Ravdin, D.E. Koppel, J. Schlessinger, W.W. Webb, E.L. Elson, and T.R. Podleski 1976 Lateral motion of fluorescently labled acetylcholine receptors in membranes of developing muscle fibers. Proc. Natl. Acad. Sci. USA, 73:4594-4598.

Bader, D. 1980 Reinnervation of motor endplate-containing and motor endplate-less muscle grafts. Dev. Biol., 77:315-327.

Bader, D. 1981 Density and distribution of alpha-bungarotoxin-binding sites in postsynaptic structures of regenerated rat skeletal muscle. J. Cell Biol., 88:338345 .

Bennett, M.R., E.M. McLachlan, and R.S. Taylor 1973 The formation of synapses in reinnervated mammalian striated muscle. J. Physiol., (Lond.), 233:481-500.

Bennett, M.R., T. Florin, and R. Woog 1974 The formation of synapses in regenerating mammlian striated muscle. J. Physiol., (Lond.), 238:79-92.
Braithwaite, A.W., and A.J. Harris 1979 Neural influence on acetylcholine receptor clusters in embryonic development of skeletal muscles. Nature, 279:549-551.

Burden, S.J., P.B. Sargent, and U.J. McMahan 1979 Acetylcholine receptors in regenerating muscle accumulate at the original synaptic sites in the absence of the nerve. J. Cell Biol., 82:412-425.

Carlson, B.M., and J.A. Faulkner 1983 The regeneration of skeletal muscle fibers following injury: A review. Med. Sci. Sports Exerc., 15:187-198.

Carlson, B.M., P. Hnik, S. Tucek, R. Vejsada, D.M. Bader, and J.A. Faulkner 1981 Comparison between grafts with intact nerves and standard free grafts of the rat extensor digitorum longus muscle. Physiol. Bohemoslov. 30:505-513.

Dennis, M.J., L. Ziskind-Conhaim, and A.J. Harris 1981 Development of neuromuscular junctions in rat embryos. Dev. Biol., 81:266-279.

Frank, E., J.K.S. Jansen, T. Lomo, and R.H. Westgaard 1975 The interaction between foreign and original motor nerves innervating the soleus muscle of rats. J. Physiol., (Lond.), 247:725-743.

Gerding, R., N. Robbins, and J. Antosiak 1977 Efficiency of reinnervation of neonatal rat muscle by original and foreign nerves. Dev. Biol., 61:177-183.

Gorio, A., G. Carmignoto, M. Finesso, P. Polato, and M.G. Nunzi 1983 Muscle reinnervation. II. Sprouting, synapse formation, and repression. Neuroscience, 8:403-416.

Gruber, H., G. Freilinger, J. Holle, and R. Mayr 1974 Motor endplates in autologous muscle transplants. Experientia, 30:1191-1192.

Hakelius, L. 1979 Free muscle grafting. Clin. Plast. Surg., 6:301-316.

Hansen-Smith, F.M. 1983 Development and innervation of soleplates in the freely grafted extensor digitorum longus muscle in the rat. Anta. Rec., 207:55-67.

Hansen-Smith, F.M., and B.M. Carlson 1979 Cellular responses to free grafting of the extensor digitorum longus muscle of the rat. J. Neurol. Sci., 41:149-173.

Harris, A.J. 1981 Embryonic growth and innervation of rat skeletal muscles. III. Neural regulation of junctional and extra-junctional acetylcholine receptor clusters. Philos. Trans R. Soc. Lond. [Biol.], 293:287-314.

Harris, J.B. 1981 Reconstruction of neuromuscular junctions in rat skeletal muscles following assault by the myotoxic neurotoxin, notexin. Br. J. Pharmacol., 74:249P-250P.

Harris, J.B. 1982 Restitution of neuromuscular transmission in regenerating muscles following assault by the myotoxic neurotoxin notexin. Toxicon, 20:116-117.

Hartzell, H.C., and D.M. Fambrough 1972 Acetylcholine receptors. Distribution and extrajunctional density in rat diaphragm after denervation correlated with acetylcholine sensitivity. J. Gen. Physiol. 60:248-262.

Ishikawa, Y., and Y. Shimada 1982 Acetylcholine receptors and cholinesterase in developing chick skeletal muscle fibers. Dev. Brain Res., 5:187-197.

Iwayama, T. 1969 Relation of regenerating nerve terminals to original endplates. Nature, 224:81-82.

Jansen, J.K.S., and D. Van Essen 1975 Reinnervation of rat skeletal muscle in the presence of alpha-bungarotoxin. J. Physiol. (Lond.), 250:651-667.

Karnovsky, M.J. 1964 The localization of cholinesterase activity in rat cardiac muscle by electron microscopy. J. Cell Biol., 23:217-231.

Kidokoro, Y., and E. Yeh 1982 Initial synaptic transmission at the growth cone in Xenopus nerve-muscle cultures. Proc. Natl. Acad. Sci. USA, 79:6727-6731.

Kidokoro, Y., M.J. Anderson, and R. Gruener 1980 Changes in synaptic potential properties during acetylcholine receptor accumulation and neurospecific in- 
teractions in Xenopus nerve-muscle cell culture. Dev. Biol., 78:464-483.

Korneliussen, H., and H. Sommerschild 1976 Ultrastructure of the new neuromuscular junctions during rein nervation of rat soleus muscle by a "foreign" nerve. Cell Tissue Res., 167:439-452.

Lomo, T., and C.R. Slater 1980 Acetylcholine sensitivity of developing ectopic nerve-muscle junctions in adult rat soleus muscle. J. Physiol. (Lond.) 303:173-189.

Luft, J.H. 1971 Ruthenium red and violet. II. Fine structural localization in animal tissues. Anat. Rec. $171: 369-416$

Marshall, L.M., J.R. Sanes, and U.J. McMahan 1977 Reinnervation of original synaptic sites on muscle fiber basement membrane after disruption of the muscle cells. Proc. Natl. Acad. Sci. USA, 74:3073-3077.

McMahan, U.J., and C.R. Slater 1984 The influence of basal lamina on the accumulation of acetylcholine receptors at synaptic sites in regenerating muscle. J. Cell Biol., 98:1453-1473.

McMahan, U.J., D.R. Edgington, and D.P. Kuffler 1980 Factors that influence regeneration of the neuromuscular junction. J. Exp. Biol., 89:31-42.

Moody-Corbett, F., and M.W. Cohen 1981 Localization of cholinesterase at sites of high acetylcholine receptor density on embryonic amphibian muscle cell cultured without nerve. J. Neurosci., 1:596-605.

Moody-Corbett, F., and M.W. Cohen 1982 Influence of nerve on the formation and survival of acetylcholine receptor and cholinesterase patches on embryonic Xenopus muscle cells in culture. J. Neurosci., 2:633-646.

Peng, H.B., and P.-C. Cheng 1982 Formation of postsynaptic specializations induced by latex beads in cultured muscle cells. J. Neurosci., 2:1760-1774.

Porter, C.W., and E.A. Barnard 1975 Distribution and density of cholinergic receptors at the motor endplates of a denervated mouse muscle. Exp. Neurol., 48:542556 .

Prives, J., I. Silman, and A. Amsterdam 1976 Appear ance and disappearance of acetylcholine receptor during differentiation of chick skeletal muscle in vitro. Cell, 7:543-550.

Ravdin, P., and D. Axelrod 1977 Fluorescent tetramethyl rhodamine derivatives of alpha-bungarotoxin: Preparation, separation and characterization. Anal Biochem, 80:585-592.

Robbins, N., J. Antosiak, R. Gerding, and O.D. Uchitel 1977 Nonacceptance of innervtion by innervated neonatal rat muscle. Dev. Biol, 61:166-176.

Sanes, J.R., L.M. Marshall, and U.J. McMahan 1978 Reinnervation of muscle fiber basal lamina after removal of myofibers. J. Cell Biol., 78:176-198.

Sanes, J.R., D.H. Feldman, J.M. Cheney, and J.C. Lawrence 1984 Brain extract induces synaptic characteristics in the basal lamina of cultured myotubes. J. Neurosci., 4:464-473.
Slater, C.R. 1982 Neural influence on the postnatal changes in acetylcholine receptor distribution at nerve muscle junctions in the mouse. Dev. Biol., 94:23-30.

Snow, M.H. 1978 An autoradiographic study of satellite cell differentiation into regenerating myotubes following transplantation of muscles in young rats. Cell Tissue Res., 186:535-540.

Spector, I., and J.M. Prives 1977 Development of electrophysiological and biochemical membrane properties during differentiation of embryonic skeletal muscle in cuiture. Proc. Natl. Acad. Sci. USA, 74:5166-5170.

Steinbach, J.H. 1981 Developmental changes in acetylcholine receptor aggregates at rat skeletal neuromuscular junctions. Dev. Biol., 84:267-276.

Thompson, N. 1971 Investigation of autogenous skeletal muscle free grafts in the dog. With a report on a suc cessful free graft of skeletal muscle in man. Transplantation, 12:353-363.

Thompson, N. 1974 A review of autogenous skeletal muscle grafts and their clinical applications. Clin. Plast. Surg., 1:349-403.

Thompson, N., A.P. Naftalin, and J.T. Lee 1978 Experimental study of the role of the motor endplate zone in the transplantation of skeletal muscle autografts in rabbits. Chir. Plast., 4:81-85.

Tsuji, S., and C. Tobin-Gros 1980 A simple silver nitrate impregnation of nerve fibers with preservation of acetylcholinesterase activity at the motor end-plate. Ex perientia, 36:1317-1319.

Vedung, S. 1983 Transplantation of Striated Muscle With Special Reference to Facial Paralysis. Ph.D. thesis, Uppsala University.

Waerhaug, O., H. Korneliussen, and H. Sommerschild 1977 Morphology of motor nerve terminals on rat soleus muscle fibers reinnervated by the original and by a "foreign" nerve. Anta. Embryol., 151:1-15.

Weinberg, C.B., C.G. Reiness, and Z.W. Hall 1981a Topographical segregation of old and new acetylcholine receptors at developing ectopic endplates in adult rat muscle. Cell Biol., 88:215-218.

Weinberg, C.B., J.R. Sanes, and Z.W. Hall 1981b Formation of neuromuscular junctions in adult rats: Accumultion of acetylcholine receptors, acetylcholinesterase, and components of synaptic basal lamina. Dev. Biol., 84:255-266.

Womble, M.D. 1982a Alpha-bungarotoxin binding site accumulation during rat skeletal muscle regeneration. Anat. Rec., 202:207A-208A (abstr.)

Womble. M.D. $1982 \mathrm{~b}$ Role of the original synaptic site on acetylcholine receptor clustering and nerve-muscle contact formation in regenerating rat skeletal muscle. Soc. Neurosci. Abstr., 8:188 (abstr.)

Ziskind-Conhaim, L., and J.I. Bennett 1982 The effects of electrical inactivity and denervation on the distri bution of acetylcholine receptors in developing rat muscle. Dev. Biol., 90:185-197. 